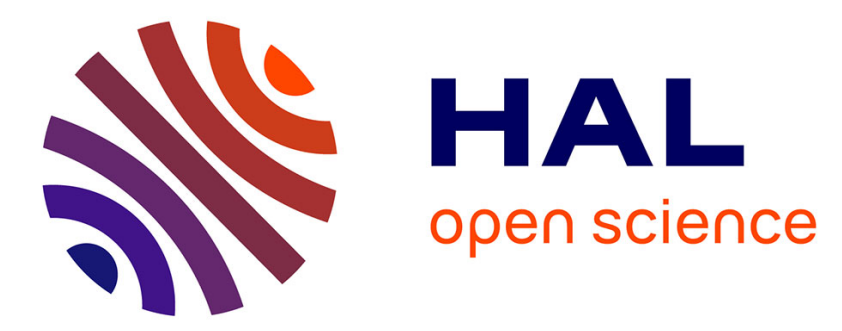

\title{
Non-Boussinesq convection in a square cavity with surface thermal radiation
}

\author{
Madiha Bouafia, Saber Hamimid, Messaoud Guellal
}

\section{To cite this version:}

Madiha Bouafia, Saber Hamimid, Messaoud Guellal. Non-Boussinesq convection in a square cavity with surface thermal radiation. International Journal of Thermal Sciences, 2015, 96, pp.236-247. 10.1016/j.ijthermalsci.2015.04.017 . hal-01178951

\section{HAL Id: hal-01178951 \\ https://hal.science/hal-01178951}

Submitted on 30 Oct 2018

HAL is a multi-disciplinary open access archive for the deposit and dissemination of scientific research documents, whether they are published or not. The documents may come from teaching and research institutions in France or abroad, or from public or private research centers.
L'archive ouverte pluridisciplinaire HAL, est destinée au dépôt et à la diffusion de documents scientifiques de niveau recherche, publiés ou non, émanant des établissements d'enseignement et de recherche français ou étrangers, des laboratoires publics ou privés. 


\title{
Non-Boussinesq convection in a square cavity with surface thermal radiation
}

\author{
M. Bouafia ${ }^{\text {a, }}{ }^{*}$, S. Hamimid ${ }^{\text {b }}$, M. Guellal ${ }^{b}$ \\ ${ }^{a}$ Laboratoire de Mécanique et Energétique d'Evry, Université d'Evry, CE 1455 Courcouronnes, 91020 Evry Cedex, France \\ ${ }^{\mathrm{b}}$ Laboratoire de Génie des Procédés Chimiques, Université de Sétif, 19000 Sétif, Algeria
}

\begin{abstract}
The interaction of natural convection with surface radiation in a differentially heated square cavity filled with air is considered under large temperature differences. The study has been investigated by direct numerical simulations with a two-dimensional finite volume numerical code solving the timedependent Navier-Stokes equations under the Low Mach Number (LMN) approximation. Calculations were performed for cases with strong non-Boussinesq effects. The results reveal that the fluid flow and heat transfer are influenced significantly by the surface radiation. At steady state, the top wall is cooled and the bottom wall is heated compared to the case without radiation. The air flow is reinforced near the horizontal walls and the thermal stratification at the core is reduced. The surface radiation reduces the convection heat transfer at the hot wall and increases it on the cold wall.

Transition from steady to unsteady flow has also been investigated. By comparing the solutions in pure convection, the results in combined convection-radiation show that the radiation promotes the occurrence of instabilities leading to an early transition to the unsteadiness and contributes to the modification of the physical mechanism responsible for their onset.
\end{abstract}

\section{Introduction}

Problems of natural convection coupled to thermal radiation are encountered in many industrial applications such as furnaces, combustion equipments, burners, cooling of electronic components, printed circuit boards.... Most of these applications proceed with large temperature differences and require a modeling which accounts for realistic fluid properties variations and compressibility effects. Derived from the compressible Navier-Stokes equations, the Low Mach Number (LMN) approximation constitutes an important numerical problem for low speed compressible flows and has the advantage that it presents the same mathematical structure as the incompressible Navier-Stokes equations. In this approximation, the total pressure is split into two terms: a mean thermodynamic pressure which is spatially uniform and depends only on time and a dynamic pressure. Since for Low Mach flows the thermodynamic pressure is very high compared to the dynamic pressure, this decomposition leads to eliminate the acoustic waves

\footnotetext{
* Corresponding author. Tel.: +3316947 75 42; fax: +33169 477599. E-mail address: bouafia@ufrst.univ-evry.fr (M. Bouafia).
}

which present a severe limitation on the time steps used for numerical integration while large variations of density with temperature are allowed. The interaction between radiation and convection in solid-fluid domains exists in a case of participating media but also by the influence of boundary conditions. The differentially heated cavity problem is a classical case commonly used in the process of CFD codes verification. The aim of this paper is to study the effect of surface thermal radiation on natural convection in a differential heated cavity under strong temperature differences.

Research in radiative-convective heat transfer was investigated in a variety of geometrical configurations and recently they are considered mainly by numerical methods. Several studies concern convection flows in transparent media bounded partially or totally by solid walls: $2 D$ or $3 D$ rectangular cavities [1-5], closed cavities with a circular or square obstruction inside [6-11], partitioned cavities with baffles [12-14], partially open cavities [15-17], vertical channels [18]. The coupling natural convection, conduction and surface thermal radiation has also been investigated in two $[19,20]$ or three-dimensional rectangular cavities [21].

Among the numerical studies cited above, the physical problem is described by the Navier-Stokes equations under the 


\begin{tabular}{|llll|}
\hline \multicolumn{2}{|l|}{ Nomenclature } & $v_{z}$ & vertical component of the velocity \\
$A$ & aspect ratio, $A=H / L$ & Greek symbols \\
$c_{p}, c_{v}$ & specific heat capacities & $\alpha$ & thermal diffusivity, $\alpha=\kappa / \rho c_{p}$ \\
$H$ & height & $\delta$ & normalized temperature difference, $\delta=\Delta T / 2 T_{0}$ \\
$L$ & width & $\kappa$ & thermal conductivity \\
$L_{0}$ & reference length & $\mu$ & dynamic viscosity \\
$N_{r}$ & radiation-conduction number, $N_{r}=\sigma T_{0}^{3} L_{0} / 2 \delta \kappa_{0}$ & $\pi$ & reduced pressure \\
$N u_{c v}$ & average convective Nusselt number & $\rho$ & density \\
$N u_{r a d}$ & average radiative Nusselt number & $\sigma$ & Stefan-Boltzmann constant \\
$N u_{g}$ & global Nusselt number, $N u_{g}=N u_{c v}+N u_{r a d}$ & $\Delta T$ & Temperature difference, $T_{h}-T_{c}$ \\
$\bar{P}$ & mean thermodynamic pressure & $\varepsilon$ & wall emissivity \\
$P_{0}$ & reference pressure, $P_{0}=\rho_{0} V_{0}^{2}$ & & \\
$P r$ & Prandtl number, $P r=\mu_{0} / \rho_{0} \alpha_{0}$ & $S u b s c r i p t s$ \\
$Q_{r}$ & dimensionless net radiative flux & 0 & values at reference temperature \\
$R a$ & Rayleigh number, $R a=2 \rho_{0} \delta g L_{0}^{3} / \mu_{0} \alpha_{0}$ & $h$ & hot \\
$t_{0}$ & reference time, $t_{0}=L_{0} / V_{0}$ & $b$ & bottom \\
$T$ & non-dimensional temperature & $c$ & cold \\
$T_{0}$ & reference temperature, $T_{0}=\left(T_{h}+T_{c}\right) / 2$ & $l$ & left \\
$V_{0}$ & reference velocity, $V_{0}=\mu_{0} R a / \rho_{0} L_{0}$ & $r$ & right \\
$v_{x}$ & horizontal component of the velocity & $t$ & top \\
& & &
\end{tabular}

Boussinesq approximation and a radiation algorithm is used for gray and diffuse surfaces. Note that the Boussinesq approximation is valid only for low temperature differences and considers all the physical properties constant except in the buoyancy force. It results from these studies that radiation influences the heat transfer substantially. For instance, Akiyama and Chong [1] studied the interaction of natural convection with radiation in a square cavity filled with air. Their results have shown that the surface radiation significantly affects the temperature distribution and the flow patterns, especially at higher Rayleigh number. The radiation heat transfer plays an important part in overall heat flux and increases with the increase of emissivity. The convection Nusselt number increases with Ra but little variations were observed with the emissivity. Colomer et al. [2] analyzed the coupling radiation and natural convection in a three-dimensional differentially heated cavity. Both transparent and participating media were explored. For transparent media, the effects of surface radiation on the heat transfer were investigated for different values of the Rayleigh number and a given Planck number. A comparison with a twodimensional case is presented showing a good agreement between both solutions. Wang et al. [3] studied numerically the interaction of convection with radiation in a two-dimensional differentially heated square cavity. It has been found that the surface radiation reduces the stratification in the cavity core and increases the average Nusselt number with increasing the emissivity. The transition from steady to unsteady flow is also investigated. The results have shown an early transition to the unsteadiness compared to the case without radiation.

Merzhab et al. [6] considered a differentially heated cavity with an inner conducting square body. They found that the radiation exchange homogenizes the temperature inside the cavity and produces an increase in the average Nusselt number as the emissivity increases. Sun et al. [7] considered a square air-filled cavity cooled from below and above, with a heated square body located at the cavity center. The flow structure is investigated for various Rayleigh numbers, emissivity of the walls and sizes of the inner body. Recently, a numerical study has been conducted by Saravanan and Sivaraj [12] in a differentially heated cavity with a heated plate placed horizontally or vertically at its center. It has been found that the convective heat transfer increases with emissivity if the plate is horizontal and decreases if the plate is vertical. The overall heat transfer by convection and radiation increases with the emissivity and the presence of radiation leads to a better homogenization of the temperature within the cavity for both positions of the central plate.

Convection-radiation coupling in the presence of large temperature variations was the purpose of few studies and concerned participating media. The numerical studies performed by Ioan Telega et al. [22], Dubroca et al. [23] and Scarella et al. [24] on various configurations are based on the LMN model. From the literature, it appears that no work was performed on coupled convection with surface radiation under large temperature differences. In this paper two-dimensional simulations under the LMN approximation are reported for a square cavity with gray and diffuse surfaces. Following the previous study of Wang et al. [3], the purpose of this investigation is to analyze the influence of surface radiation on the flow pattern and heat transfer and on the transition from steady to unsteady flows for strong non-Boussinesq regimes. The code validation is performed by comparison with the well-documented results in literature.

\section{Problem formulation}

\subsection{Governing equations}

The problem considered here is natural convection in a square cavity placed into a gravitational field $\vec{g}$ which is parallel to the active walls. The inner surfaces are assumed to be diffuse, gray and opaque. The left and right side walls are isothermal at respective temperatures $T_{h}$ and $T_{c}\left(T_{c}<T_{h}\right)$, and the bottom and top walls are adiabatic. The cavity is filled with a transparent gas initially at a uniform temperature $T_{0}=\left(T_{h}+T_{c}\right) / 2$ and pressure $P_{0}$. It is assumed to be an ideal gas with constant specific heat capacities $c_{p}$ and $c_{v}$ of ratio $\gamma=1.4$. Its dynamic viscosity $\mu$ and thermal conductivity $\kappa$ are allowed to depend on temperature. As we are interested in flows induced by large temperature differences, the problem is governed by the Low Mach approximation equations to describe such a flow (Paolucci [25]). The equations are made dimensionless by reference quantities: $L_{0}=L, t_{0}=L_{0} / V_{0}, T_{0}=\left(T_{h}+T_{c}\right) / 2$, $P_{0}=\rho_{0} V_{0}^{2}, V_{0}=\mu_{0} / \rho_{0} L_{0} R a$. The physical properties (density, 
dynamic viscosity, thermal conductivity, thermal diffusivity) are scaled by $\rho_{0}, \mu_{0}, \kappa_{0}, \alpha_{0}$ where the subscript 0 denotes values at the reference temperature $T_{0}$. The governing equations in the dimensionless form are given by Bouafia and Daube [26]:

$\frac{\partial \rho}{\partial t}+\nabla \cdot(\rho \boldsymbol{V})=0$

$\rho\left(\frac{\partial \boldsymbol{V}}{\partial t}+(\boldsymbol{V} \cdot \nabla) \boldsymbol{V}\right)=-\nabla \Pi+(R a)^{-1 / 2} \nabla \cdot \overline{\bar{\tau}}-\operatorname{Pr}^{-1} \frac{\rho-1}{2 \delta} \boldsymbol{z}$

$\rho\left(\frac{\partial T}{\partial t}+(\boldsymbol{V} \cdot \nabla) T\right)=\frac{1}{\operatorname{Pr} R a^{1 / 2}} \nabla \cdot(\kappa \nabla T)+\frac{\gamma-1}{\gamma} \frac{d \bar{P}}{d t}$

$\bar{P}=\rho T$

$\boldsymbol{V}$ is the velocity vector of components $\left(v_{x}, v_{z}\right)$, $\overline{\bar{\tau}}=\mu\left(\nabla \boldsymbol{V}+(\nabla \boldsymbol{V})^{t}-2 / 3(\nabla \cdot \boldsymbol{V}) \overline{\bar{I}}\right)$ the viscous stress tensor, $\Pi=(P-$ $\left.\bar{P}+\rho_{0} g z\right) / \rho_{0} V_{0}^{2}$ the reduced pressure, $\delta=\Delta T / 2 T_{0}$ the normalized temperature difference with $\Delta T=\left(T_{h}-T_{c}\right)$ and $\bar{P}$ the mean thermodynamic pressure. $\mathrm{d} \overline{\mathrm{P}} / \mathrm{dt}$ is calculated by integrating on the whole domain $\Omega$ the energy equation and taking into account the continuity equation:

$\frac{d \bar{P}}{d t}=\frac{\gamma}{A P r R a^{1 / 2}} \int_{\Gamma} \kappa \frac{\partial T}{\partial \mathbf{n}} d l$

The independent dimensionless parameters are the aspect ratio $A=H / L$ equal to 1 in this study, the Prandtl number and the Rayleigh number defined at the reference temperature $T_{0}$ :

$\operatorname{Pr}=\frac{\mu_{0}}{\rho_{0} \alpha_{0}} ; \quad R a=\frac{2 \rho_{0} \delta g L_{0}^{3}}{\mu_{0} \alpha_{0}}$

where $\alpha_{0}=\kappa_{0} / \rho_{0} c_{p}$ is the thermal diffusivity. The system of equations is closed by Sutherland's law for the dynamic viscosity $\mu$ :

$\mu(T)=T^{3 / 2} \frac{1+S_{\mu}}{T+S_{\mu}}$

$S_{\mu}=110.4 K / T_{0}$ for air at standard conditions. Since the Prandtl number is assumed to be constant and the influence of the temperature on $c_{p}$ is neglected, we obtain $\tilde{\mu} / \mu_{0}=\tilde{\kappa} / \kappa_{0}$ and then the thermal conductivity is given by $\kappa(T)=\mu(T)$. Tilde refers to dimensional quantities.

\subsection{Boundary conditions}

At the boundaries, no-slip conditions for velocities are added to thermal conditions. By considering the surface radiation, the adiabatic conditions on horizontal walls imply that convection flux balances net radiative flux.

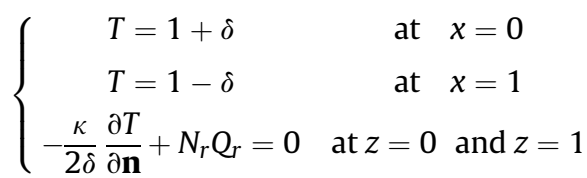

$\mathbf{n}$ is the normal vector inward the cavity and $N_{r}$ the radiation-conduction number (inverse of the Planck number) given by:

$N r=\frac{\sigma T_{0}^{4} L_{0}}{\kappa_{0}\left(T_{h}-T_{C}\right)}=\frac{\sigma T_{0}^{3} L_{0}}{2 \delta \kappa_{0}}$

$\sigma$ is the Stephan-Boltzmann constant and $Q_{r}=q_{r} / \sigma T_{0}^{4}$ the dimensionless net radiative heat flux. This quantity is determined from a radiation analysis based on the radiosity method.

\subsection{Discrete radiation equations}

For non participating media, the thermal radiation equation is solved using the radiosity method for diffuse-gray surfaces. The walls are divided in elements according to the mesh used for the governing equations. The radiosity equation for the $i^{\text {th }}$ element is given by:

$J_{i}=\varepsilon_{i} T_{i}^{4}+\left(1-\varepsilon_{i}\right) \sum_{j=1}^{N} F_{i-j} J_{j}$

where $J_{i}$ is the non-dimensional radiosity of the corresponding element, the first term on the right hand is the emitted power by the surface $i$ and $\left(1-\varepsilon_{i}\right) \sum_{j=1}^{N} F_{i-j} J_{j}$ the reflected radiant energy coming from surrounding surfaces that can be seen by the surface $i$. $\varepsilon_{i}$ is the emissivity of the element, $F_{i-j}$ the geometry view factor from the $i^{t h}$ element to the $j$ th and $\mathrm{N}$ the total number of elements along the walls of the cavity. View factors $F_{i-j}$ are calculated using analytical expressions according to the relative position between surfaces [27]. The radiosity equation can be reduced to a simple linear system $a_{i j} j_{j}=b_{i}$ :

$\sum_{j=1}^{N}\left(\delta_{i j}-\left(1-\varepsilon_{i}\right) F_{i-j}\right) J_{j}=\varepsilon_{i} T_{i}^{4}$

where $a_{i j}=\delta_{i j}-\left(1-\varepsilon_{i}\right) F_{i-j}$ and $b_{i}=\varepsilon_{i} T_{i}^{4}$. The dimensionless net radiative heat flux $Q_{r}$ leaving the $i$ th element surface is obtained from equation:

$Q_{r, i}=J_{i}-\sum_{j=1}^{N} F_{i-j} J_{j}$

\subsection{Heat transfer}

The average Nusselt numbers characterizing the contributions of convection and thermal radiation through the vertical walls, are respectively defined as:

$N u_{c v}=\frac{1}{2 A \delta} \int_{0}^{A}-\kappa \frac{\partial T}{\partial \mathbf{n}} d z ; \quad N u_{\text {rad }}=\frac{1}{A} \int_{0}^{A} N_{r} Q_{r} d z$

The global average Nusselt number is given by: $N u_{g}=N u_{c v}+N u_{\text {rad }}$.

\subsection{Numerical modeling}

The $2 D$ governing equations are solved numerically by a finitevolume scheme using the staggered arrangement. Equations (1)-(4) were discretized in time by a second-order backward Euler scheme in which the diffusive and viscous linear terms are implicitly treated while the convective nonlinear terms are explicitly treated using an Adams-Bashforth extrapolation. Since the equations are of incompressible type, the velocity-pressure coupling is handled by a technique derived from the classical projection method. The spatial discretization applied is based on a uniform grid in the vertical direction and a non-uniform grid in the horizontal direction refined in the vicinity of the vertical walls by using Chebychev collocation points. The Poisson equations for the pressure correction in the projection method are solved by 
Table 1

Average Nusselt number at the vertical walls and normalized pressure in pure convection for $\delta=0.6$ and $T_{0}=600 \mathrm{~K}$.

\begin{tabular}{lllrrl}
\hline & $\mathrm{Ra}$ & $\mu$ & \multicolumn{1}{c}{$N u_{h}$} & \multicolumn{1}{c}{$N u_{c}$} & \multicolumn{1}{l}{$P / P_{0}$} \\
\hline Present & $10^{6}$ & Sutherland's law & 8.6883 & 8.8683 & 0.924436 \\
Le Quéré et al. [28] & $10^{6}$ & Sutherland's law & 8.6866 & 8.6866 & 0.924487 \\
Present & $10^{7}$ & Sutherland's law & 16.2462 & 16.2462 & 0.922547 \\
Le Quéré et al. [28] & $10^{7}$ & Sutherland's law & 16.2410 & 16.2410 & 0.92263 \\
\hline
\end{tabular}

standard multigrid techniques. A detailed description of the algorithm is found in Ref. [26]. For thermal boundary conditions (Eq. (8)), an explicit assessment of $Q_{r}$ is applied leading to the following equations at time level $n+1$ :

$\left\{\begin{array}{cl}-\frac{\kappa}{2 \delta} \frac{\partial T^{n+1}}{\partial z}+N_{r}\left(2 Q_{r}^{n}-Q_{r}^{n-1}\right)=0 & \text { at } \quad z=0 \\ \frac{\kappa}{2 \delta} \frac{\partial T^{n+1}}{\partial z}+N_{r}\left(2 Q_{r}^{n}-Q_{r}^{n-1}\right)=0 & \text { at } z=1\end{array}\right.$

This treatment allows us to obtain first the temperature $T^{n+1}$ by solving the Navier-Stokes equations and obtain the temperature on the insulated boundaries, then $Q_{r}^{n+1}$ by solving the radiosity equation (Eq. (11)) with the Gauss elimination method.

\subsection{Code validation}

The non-Boussinesq code (NBC) was first validated against a benchmark solution [28] corresponding to convection without radiation in a differentially heated square cavity. Tests have been carried out for a normalized temperature difference $\delta=0.6$ at $R a=10^{6}$ and $10^{7}$ with a reference temperature $T_{0}=\left(T_{h}+T_{c}\right) /$ $2=600 \mathrm{~K}$ and variable properties. Our calculations were performed on a $257 \times 257$ non-uniform mesh refined at the walls. A good agreement is observed from Table 1 with the reference solutions based on $\mathrm{Nu}$ and the normalized thermodynamic pressure $\bar{P} / P_{0}$ resulting from this benchmark.

The code validation to predict the interaction between surface radiation and natural convection was done on a Boussinesq code (BC) and the results were compared with those obtained by Wang et al. [3]. Calculations have been carried out with the following parameters: $R a=10^{6}, \Delta T=10 \mathrm{~K}$ and a reference temperature $T_{0}=293.5 \mathrm{~K}$. The radiative number $N_{r}$ was calculated by taking $H=L=0.097 \mathrm{~m}$. Note that in order to validate the non-Boussinesq code, calculations were performed for $\varepsilon=0.017$ corresponding to $\Delta T=10 \mathrm{~K}$. As shown in Table 2, the results in term of convective and radiative Nusselt number reveal a good agreement with Wang et al. [3]. A mesh sensitivity analysis (but not presented here) was done on radiative heat transfer for two grids $129 \times 129$ and $257 \times 257$, have shown independent results of grid size. In what follows, the computations are performed with a $257 \times 257$ non-uniform grid.

\section{Numerical results}

In the LMN approximation, the governing parameters are $R a, P r$, the aspect ratio $A$ and the normalized temperature difference $\delta=\Delta T / 2 T_{0}$. By considering the radiation, other parameters are needed as the width $L$ and the wall emissivity $\varepsilon$ to determine the radiation number $N_{r}$ and the dimensionless net radiative flux $Q_{r}$. A quite extensive analysis would be necessary to cover the possible effects of each parameters. In the present study, we focused in interaction of surface radiation with strong non-Boussinesq effects at steady and unsteady states. For the steady case, the main control parameter is the wall emissivity while the following parameters are maintained constant: $A=1$ and $\delta=0.6$ which corresponds to $\Delta T=720 \mathrm{~K}$. The Prandtl number is maintained at its value for air at $300 \mathrm{~K}$, i.e. $\operatorname{Pr}=0.71$. The Rayleigh number is fixed to $R a=10^{6}$ which induces a value of the cavity length $L=0.067 \mathrm{~m}$ and a radiation number $N_{r}=16.36$. For the purpose of comparison, the numerical solutions are performed for air at initial state which is similar to that used in the 2D benchmark problem [28]: $P_{0}=101325 \mathrm{~Pa}$, $T_{0}=600 \mathrm{~K}, \rho_{0}=P_{0} /\left(r T_{0}\right)$ and $v_{x}(x, z)=v_{z}(x, z)=0$.

\subsection{Steady flow}

\subsubsection{Temperature and flow fields}

Typical isotherms and streamlines are displayed in Figs. 1 and 2 for different values of wall emissivity supposed identical on all faces. Without radiation $\varepsilon=0$, the structure of temperature fields shows patterns with thermal boundary layers growing along the vertical walls and a stratified core region. The contour lines are particularly characterized by the absence of the centro-symmetry property due to compressibility effects $\left(\left(v_{x}, v_{z}, T\right)=-\left(v_{x}, v_{z}, T\right)(1-x, 1-z)\right)$. The associated streamlines are more dense close to the vertical walls and display a main peripheral flow including asymmetrical small rolls shifted to the vertical walls.

The influence of surface radiation on the flow structure and the temperature distribution is clearly observed. In addition to asymmetrical character reinforced by the presence of surface radiation, the most visible effects occur along the horizontal walls. Compared to the case without radiation, the temperature contours clearly show the existence of temperature gradients near the top and bottom walls due to radiative exchanges and the formation of horizontal thermal boundary layers. Along the vertical walls, the isotherms are tightened on the cold wall and are more spaced on the hot wall with increasing $\varepsilon$. This behavior indicates the dominance of convective heat transfer between the fluid and the cold vertical surface. In the stratified region, the horizontal lines are more widely spaced as the emissivity increases. The corresponding streamlines highlight a different pattern at the core compared to the case without radiation. At the bottom right corner, it can be seen the streamlines which are smoothly parallel to the horizontal wall while a slight detached flow is observed in pure convection.

Table 2

Convection, radiation and global Nusselt numbers on the vertical walls; results for $R a=10^{6}, \Delta T=10 \mathrm{~K}, L_{0}=0.097 \mathrm{~m}$ and $T_{0}=293.5 \mathrm{~K}$

\begin{tabular}{|c|c|c|c|c|c|c|c|c|}
\hline & \multirow[t]{2}{*}{ Mesh } & \multirow[t]{2}{*}{$\varepsilon$} & \multicolumn{3}{|c|}{ Hot wall } & \multicolumn{3}{|c|}{ Cold wall } \\
\hline & & & $N u_{c v}$ & $N u_{\text {rad }}$ & $N u_{g}$ & $N u_{c v}$ & $N u_{\text {rad }}$ & $N u_{g}$ \\
\hline Present BC & $129 \times 129$ & 0.0 & 8.830 & 0 & 8.830 & 8.830 & 0 & 8.830 \\
\hline Present BC & $257 \times 257$ & 0.0 & 8.826 & 0 & 8.826 & 8.826 & 0 & 8.826 \\
\hline Present NBC & $129 \times 129$ & 0.0 & 8.830 & 0 & 8.830 & 8.830 & 0 & 8.830 \\
\hline Le Quéré [29] & Spectral & 0.0 & 8.825 & 0 & 8.825 & 8.825 & 0 & 8.825 \\
\hline Wang et al. [3] & N.A & 0.0 & 8.852 & 0 & 8.852 & 8.852 & 0 & 8.852 \\
\hline Saravanan et al. [12] & N.A & 0.0 & 8.827 & 0 & 8.827 & 8.827 & 0 & 8.827 \\
\hline Present BC & $257 \times 257$ & 0.2 & 8.338 & 2.355 & 10.693 & 8.374 & 2.319 & 10.693 \\
\hline Wang et al. [3] & N.A & 0.2 & 8.381 & 2.355 & 10.736 & 8.417 & 2.319 & 10.736 \\
\hline Saravanan et al. [12] & N.A & 0.2 & & & 10.671 & & & 10.671 \\
\hline
\end{tabular}




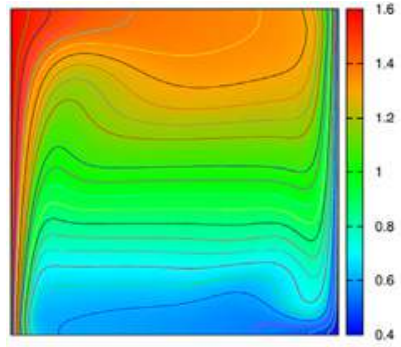

(a) $\varepsilon=0$

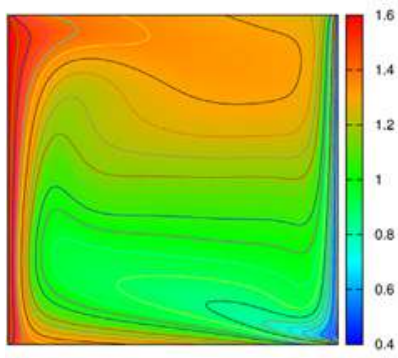

(c) $\varepsilon=0.6$

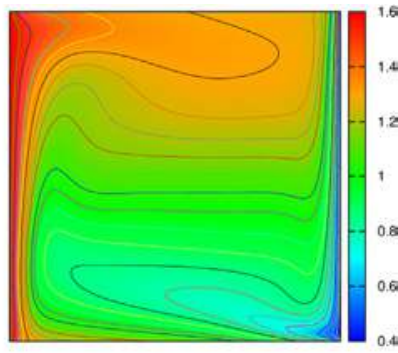

(b) $\varepsilon=0.2$

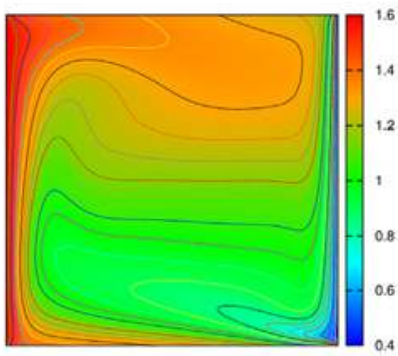

(d) $\varepsilon=0.8$
Fig. 1. Isotherms for different wall emissivities, $R a=10^{6}$ and $\delta=0.6$.

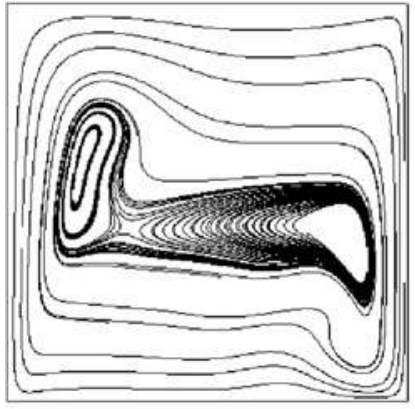

(a) $\varepsilon=0$

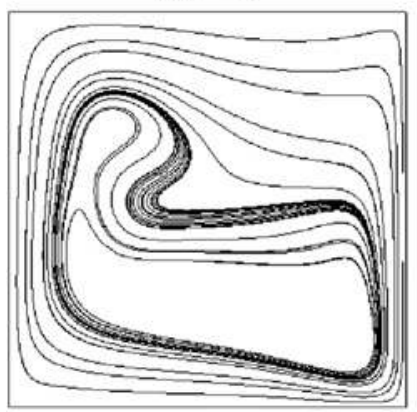

(c) $\varepsilon=0.6$

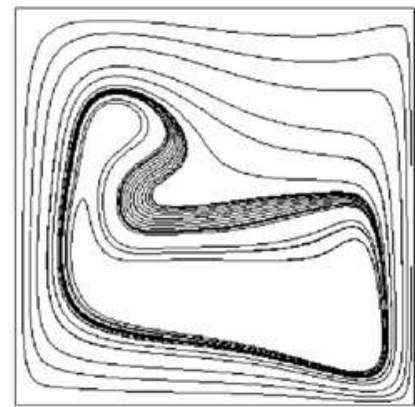

(b) $\varepsilon=0.2$

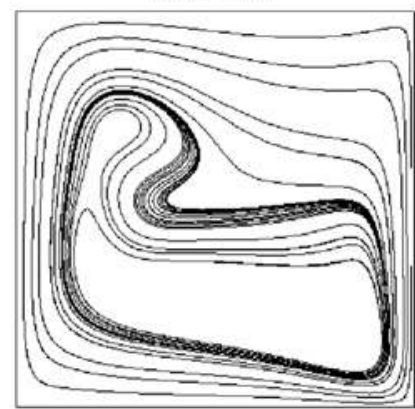

(d) $\varepsilon=0.8$
Fig. 2. Streamlines for different wall emissivities, $R a=10^{6}$ and $\delta=0.6$.

Surface radiation changes substantially the boundary conditions which significantly affect the temperature distribution and the flow structure. In Fig. 3(a), (b), are plotted the temperature profiles at the horizontal walls in both cases without and with radiation $\varepsilon=0$, $\varepsilon=0.2, \varepsilon=1$. In addition to the centro-symmetry breaking which is clearly increased by the radiation effect, note the cooling of the top wall and the heating of the bottom wall. This behavior is explained by the fact that the upper wall loses heat while the bottom wall gets heated. Indeed, Fig. 3(c), (d) shows that the net radiative flux is essentially positive at the top wall and essentially negative at the bottom wall. By comparing the cases $\varepsilon=0.2$ and $\varepsilon=1$, it can be seen that the temperature difference between the top and bottom walls decreases with increasing the wall emissivity.

It is interesting to note that the bottom wall is more affected by the wall radiation than the top wall. While the upper wall temperature slightly varies, the temperature of the bottom wall continues to increase with increasing $\varepsilon$ and tends to be close to that of the top wall for $\varepsilon=1$. For black walls, the net radiative heat flux at the bottom wall changes significantly and becomes strongly negative while at the top wall it remains almost unchanged. The asymmetric role that plays the wall radiation on the distribution of radiative heat flux and consequently on the temperature profiles explains the remarkable difference on the spatial distribution of temperature in the vicinity of horizontal walls.

Fig. 4 displays the temperature profiles along the vertical midplane and at the mid-height of the cavity. As can be seen the surface radiation induces the heating of air at the cavity core and reduces the stratification with increasing the emissivity. Fig. 3(c), (d) showing the net radiative heat flux at the horizontal walls helps to understand how the wall radiation decreases the thermal stratification. Owing to the positive flux at the top wall and negative flux at the bottom wall, the fluid is cooled (slightly) through the upper wall then heated (strongly) near the bottom wall. It results a decrease of the temperature difference between the ceiling and the floor which tends to reduce the thermal stratification. The influence of surface radiation on the velocity is illustrated in Fig. 5 were profiles of components $v_{x}$ and $v_{z}$ are plotted at $x=0.5$ and $z=0.5$ respectively. A careful look to these plots reveals that the radiation intensifies the flow near the horizontal walls as the emissivity increases. However, the radiation effect on the $v_{z}$ velocity remains negligible. Recall that the asymmetrical profiles of the vertical component $v_{z}$ result from nonlinear variations of density and transport coefficients.

\subsubsection{Wall heat transfer}

Table 3 sums the results of mean convective and radiative Nusselt numbers on the vertical walls for different wall emissivities varying from 0 to 1 . Calculations were performed with the same value $\varepsilon$ on the four walls. A closer look at these results highlights the difference between the convective and radiative heat transfer when $\varepsilon$ is different from zero. Values of convective Nusselt number are higher on the cold wall than on the hot wall while the radiative Nusselt number exhibits the opposite evolution. On the hot wall, the contribution of radiation to the global heat transfer is about $67 \%$ for $\varepsilon=0.2$ and attains $93 \%$ for $\varepsilon=1$. On the cold wall, the radiative heat transfer remains substantial with a contribution which increases from $49 \%(\varepsilon=0.2)$ to $86 \%(\varepsilon=1)$. The total Nusselt number of convection and radiation increases with the wall emissivity and attains its maximum for four black walls.

In Table 3, note that the discrepancies between the total Nusselt numbers does not exceed $0.3 \%$. The main error source comes from nonlinear boundary conditions imposed on the horizontal walls (Eq. (8)). The number of iterations required to solve the fluid equations coupled to the radiosity ones through the boundary equations is important for $\delta=0.6$. Further calculations but not shown here, were carried out to explore the influence of the parameter $\delta$ on the results accuracy. The results obtained for $\delta=0.05, \delta=0.2$ and $\delta=0.4$ revealed that the discrepancies between the total Nusselt number on the left and right sides decrease or disappear when low values of non-dimensional temperature differences are considered. For instance, the results in Table 2 confirm this tendency. Further, In Table 4, the same values at the vertical walls are obtained for a cavity with reflecting horizontal walls. 


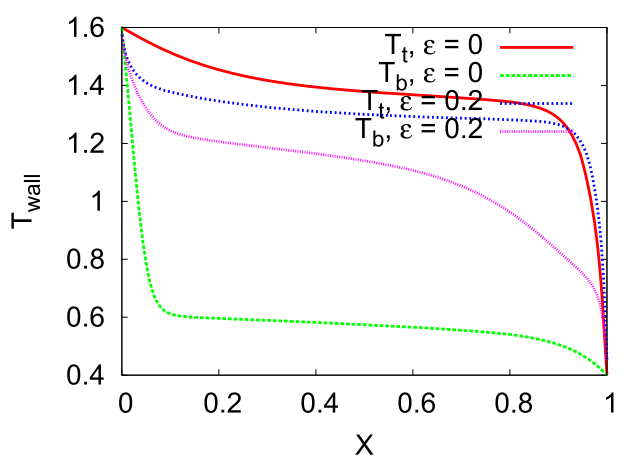

(a)

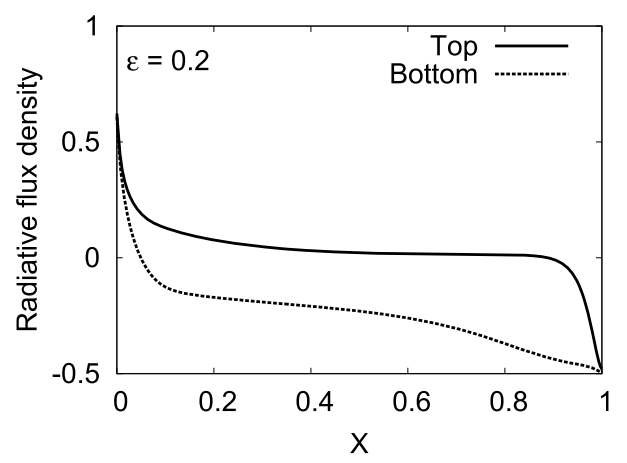

(c)

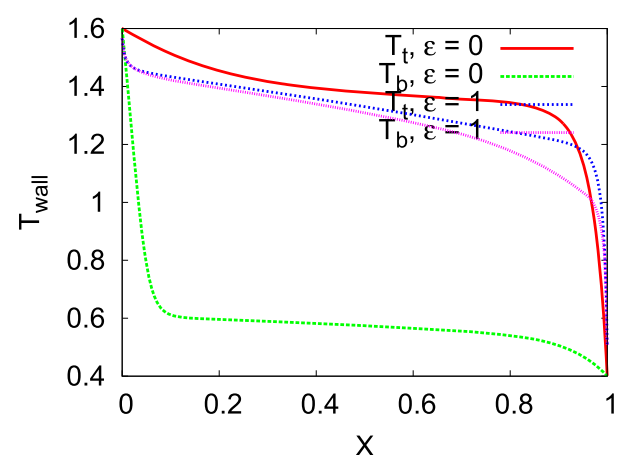

(b)

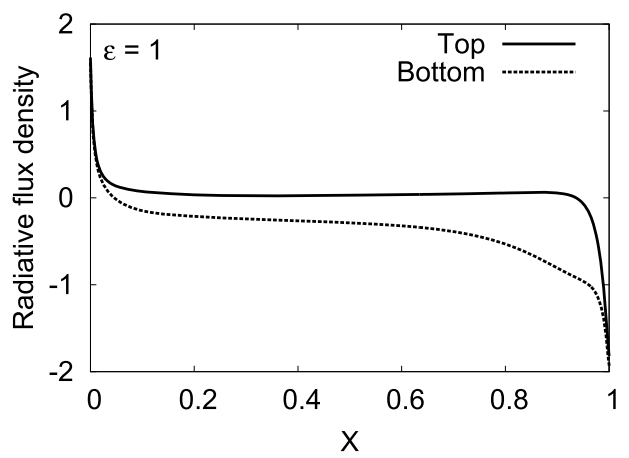

(d)

Fig. 3. (a, b) Temperature profiles and (c, d) net radiative heat flux at the horizontal walls, $R a=10^{6}$.

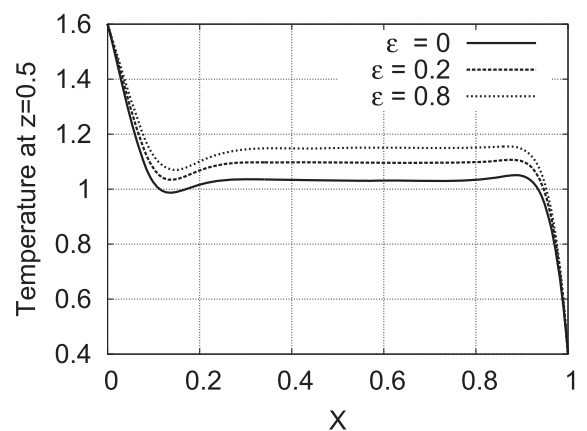

(a)

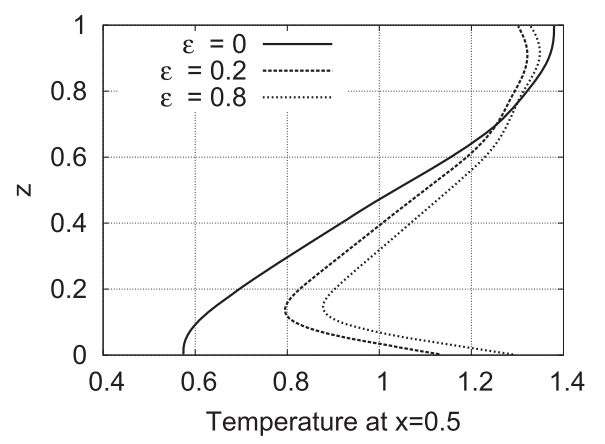

(b)

Fig. 4. Temperature profiles at mid-height $z=0.5$ and at vertical mid-plane $x=0.5, R a=10^{6}$.

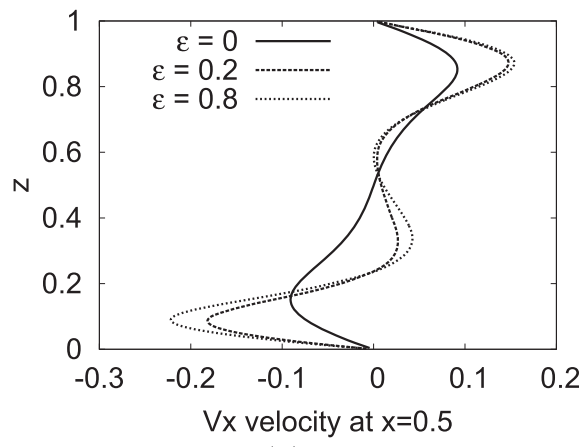

(a)

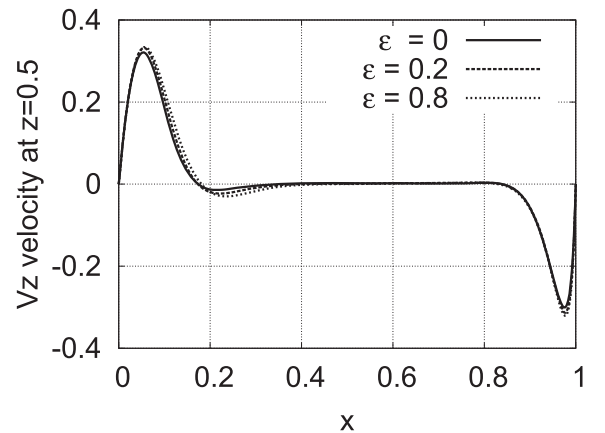

(b)

Fig. 5. Profiles of velocity components for different values of wall emissivity at $R a=10^{6}$ : (a) $v_{x}$ at $x=0.5$, (b) $v_{z}$ at $z=0.5$. 
Table 3

Convective, radiative and global Nusselt numbers on the vertical walls for different emissivities at $R a=10^{6}$; results for $\delta=0.6, T_{0}=600 \mathrm{~K}$ and $L_{0}=0.067 \mathrm{~m}$.

\begin{tabular}{|c|c|c|c|c|c|c|}
\hline \multirow[t]{2}{*}{$\varepsilon$} & \multicolumn{3}{|c|}{ Hot wall } & \multicolumn{3}{|l|}{ Cold wall } \\
\hline & $N u_{c v}$ & $N u_{\text {rad }}$ & $N u_{g}$ & $N u_{c v}$ & $N u_{\text {rad }}$ & $N u_{g}$ \\
\hline 0 & 8.6883 & 0 & 8.6883 & 8.6883 & 0 & 8.6883 \\
\hline 0.2 & 6.4614 & 12.9123 & 19.3737 & 9.8993 & 9.5203 & 19.4196 \\
\hline 0.4 & 5.9127 & 25.9921 & 31.9138 & 10.5489 & 21.4425 & 31.9914 \\
\hline 0.6 & 5.6778 & 40.3771 & 46.0549 & 10.8675 & 35.2842 & 46.1517 \\
\hline 0.8 & 5.5201 & 56.6219 & 62.1420 & 11.0206 & 51.2242 & 62.2448 \\
\hline 1 & 5.3984 & 75.2478 & 80.6463 & 11.06109 & 69.6708 & 80.7317 \\
\hline
\end{tabular}

Table 4

Convection, radiation and global Nusselt numbers on the vertical walls; configurations with black vertical walls and different wall emissivity on the horizontal walls, $R a=10^{6}$.

\begin{tabular}{|c|c|c|c|c|c|c|c|c|c|}
\hline \multirow[t]{2}{*}{$\varepsilon_{l}$} & \multirow[t]{2}{*}{$\varepsilon_{r}$} & \multirow[t]{2}{*}{$\varepsilon_{t}$} & \multirow[t]{2}{*}{$\varepsilon_{b}$} & \multicolumn{3}{|c|}{ Hot wall } & \multicolumn{3}{|l|}{ Cold wall } \\
\hline & & & & $N u_{c v}$ & $N u_{\text {rad }}$ & $N u_{g}$ & $N u_{c v}$ & $N u_{\text {rad }}$ & $N u_{g}$ \\
\hline 1 & 1 & 0 & 0 & 8.6883 & 73.0857 & 81.7740 & 8.6883 & 73.0857 & 81.7740 \\
\hline 1 & 1 & 0 & 1 & 5.0560 & 75.5920 & 80.6480 & 11.0897 & 69.6260 & 80.7157 \\
\hline 1 & 1 & 1 & 0 & 8.9807 & 72.9406 & 81.9213 & 9.0255 & 72.9157 & 81.9412 \\
\hline 1 & 1 & 1 & 1 & 5.3984 & 75.2478 & 80.6462 & 11.06108 & 69.6708 & 80.7316 \\
\hline
\end{tabular}

Fig. 6(a) highlights the opposite tendencies for the convective Nusselt number on the active walls: an increase on the cold wall with increasing $\varepsilon$ and a decrease on the hot wall with predominance values on the cold wall. For radiative heat transfer, Fig. 6(b) presents a similar behavior for both walls: an increase of the Nusselt number with emissivity but with higher values on the hot wall. In order to explain the evolution of mean convective Nusselt numbers with emissivity, it is interesting to examine the variations of local Nusselt number along the vertical walls. In Fig. 7, the average Nusselt number on the hot wall is clearly lower in the case with radiation than that obtained in the case without radiation. The most difference in the plots is observed at the leading edge $(z=0)$ and at the top. When the radiation is taken into consideration, the fluid in contact with the floor arrives heated at the leading edge. It follows a reduced temperature difference between the fluid and the wall and thus a lower heat exchange coefficient. The low level of heat transfer is maintained on a large height of the wall, except in the vicinity of the top area where the trend is reversed. The more the wall emissivity increases, the more the temperature difference between the fluid and the wall is low. This behavior tends to reduce the average convective Nusselt number on the cold wall with increasing the wall emissivity.

Fig. 8 depicts the variation of convective Nusselt number along the opposite wall for the same values of $\varepsilon$. The corresponding plots

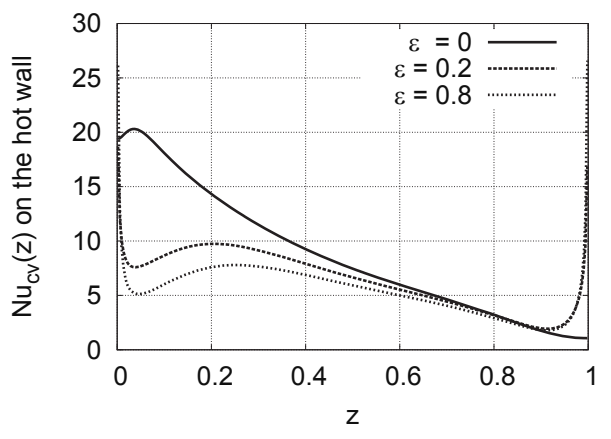

Fig. 7. Variation of local convective Nusselt number on the hot wall for different emissivities.

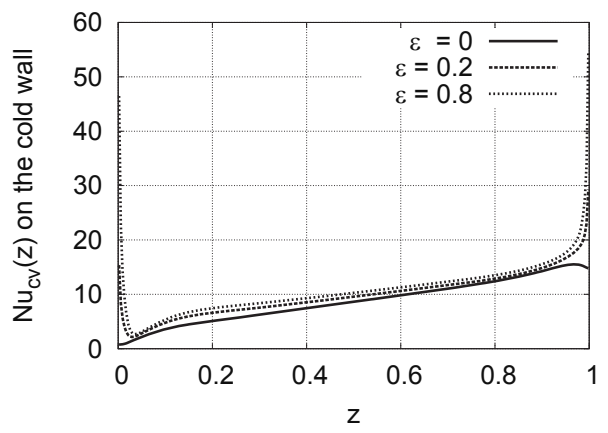

Fig. 8. Variation of local convective Nusselt number on the cold wall for different emissivities.

exhibit small areas affected by the surface radiation in the immediate vicinity of the leading edge $(z=1)$ and the bottom wall. In these restricted regions, the local Nusselt number show larger values when the radiation is taken into consideration. However, it remains almost unchanged on a large distance far from edges. By considering the mean values, the convective Nusselt number on the cold wall increases with increasing the wall emissivity $\varepsilon$. The plots related to local radiative Nusselt number on the vertical walls are shown in Fig. 9 for a large value of emissivity $\varepsilon=0.8$. Although the walls are isotherms, it can be seen that the profiles are not uniform and the maximum values are reached at almost mid-height. The plots also highlight that the average radiative Nusselt number is greater on the hot wall than on the cold wall.

In order to explore the influence of horizontal walls on the heat transfer, further calculations were carried out by testing different values of emissivity at top and bottom walls. For this purpose, the

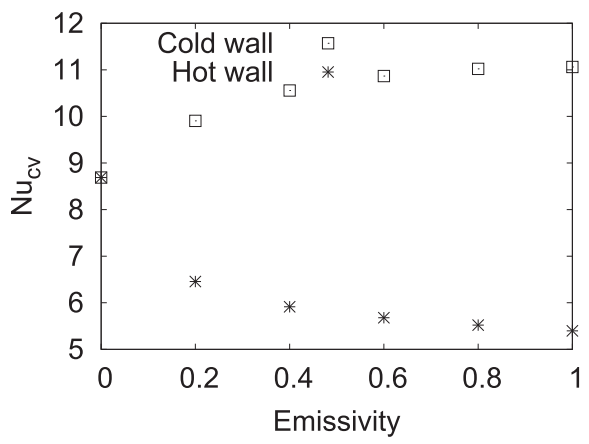

(a)

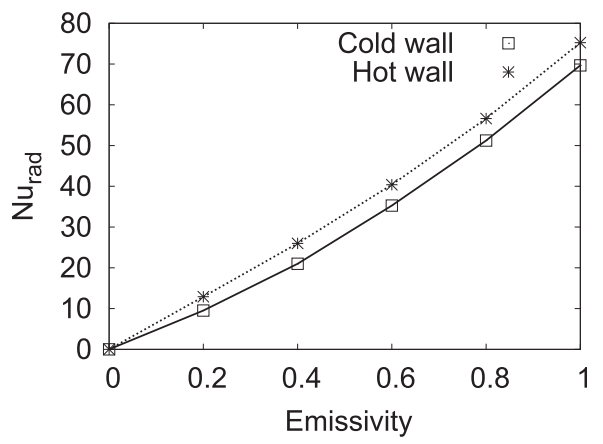

(b)

Fig. 6. Variation of average Nusselt numbers with emissivity on the vertical walls at $R a=10^{6}$; (a) convective Nusselt number, (b) radiative Nusselt number. 


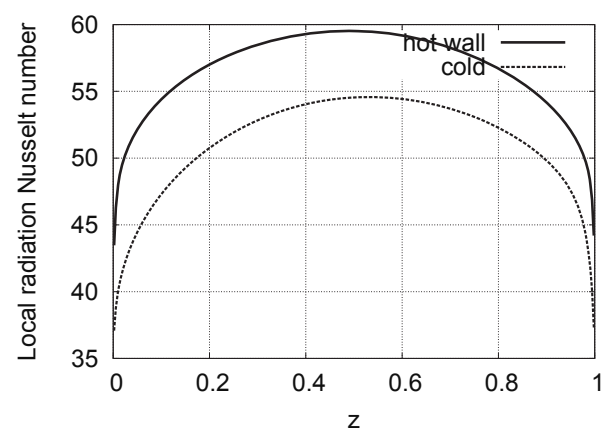

Fig. 9. Variation of local radiative Nusselt number on the vertical walls for $\varepsilon=0.8$.

vertical walls are considered black while two values $\varepsilon=0$ and $\varepsilon=1$ are imposed on the horizontal walls to obtain extreme conditions for surface thermal radiation. Table 4 summarizes the studied four cases and reveals that the total Nusselt number on the vertical walls is higher with a reflected bottom wall. The configuration $\left(\varepsilon_{t}=0\right.$, $\varepsilon_{b}=1, \varepsilon_{l}=1, \varepsilon_{r}=1$ ) gives results equivalent to those obtained with four black walls since the net radiative heat flux at the upper wall is almost equal to zero for $\varepsilon_{t}=1$. Note finally that these two configurations provide the lowest rate of heat transfer including the convective and radiative heat exchanges.

\subsection{Unsteady periodic flow}

\subsubsection{Onset of unsteadiness and nature of the bifurcation}

In this section, we are interested in the initial phase of transition to unsteady flow. In order to examine the radiation effect on the instability mechanisms, we first investigate the problem of natural convection without radiation. For $P r=0.71$ and a fixed aspect ratio $A=1$, the transition to unsteadiness in pure convection depends on the Rayleigh number and the parameter $\delta$. As we are interested in strong non-Boussinesq flow, the dimensionless temperature difference is set to $\delta=0.6$.

The procedure adopted here consists in increasing the values of Rayleigh number from a steady state until the flow becomes unsteady. Its temporal characteristics are then analyzed from time series of temperature (or velocity) at selected monitoring points. Starting from $R a=10^{6}$ and gradually increasing the Rayleigh number, the flow loses its stability beyond a critical value yielding an asymptotic periodic solution. By following this procedure up to $5 \times 10^{7}$, the bifurcation point occurs in the range $\left[3.8 \times 10^{7}\right.$; $\left.4 \times 10^{7}\right]$. Since the amplitude of periodic solutions evolves like $\left(R a-R a_{c}\right)^{0.5}$ as for a supercritical Hopf bifurcation, the method followed to determine the bifurcation point is that presented in Ref. [31]. The critical value is obtained from a linear extrapolation of the squared amplitude. The transition appears to be a supercritical Hopf bifurcation (without hysteresis effect) whose threshold lies at a critical value $R a_{c}=3.9 \times 10^{7}$ (see Fig. 10(a)). Note this value is slightly larger than that one obtained by Weissman et al. [30] $\left(R a_{c} \simeq 3.3 \times 10^{7}\right.$ for $\left.\delta=0.6\right)$ in a larger cavity $(A=2)$ which is less stabilizing. It is also lower than the critical value in the Boussinesq approximation $\left(R a_{c}=1.8 \times 10^{8}\right)$ [31]

The investigation is now extended to the convection coupled with surface radiation. In this case, the transition to unsteadiness depends on the Rayleigh number and the size of the cavity length $L$. The non-dimensional temperature difference is maintained unchanged $\delta=0.6$. The value $L$ retained in this study is deduced from the Rayleigh number $R a=5 \times 10^{7}$ corresponding to a periodic solution in pure convection (see Fig. 10(a)). Therefore, the value of the cavity length is equal to $L=0.247 \mathrm{~m}$ which leads to a radiative number $N_{r}=60.31$ (or Planck number $=0.016$ ). It should be noted that the variation of Ra depends therefore only on the variation of physical properties of air at the initial state since the parameters $L$ and $\delta$ are fixed. Recall that each solution obtained at a given Ra is used as an initial condition for a subsequent computation at a larger value of $R a$. From a practical standpoint, the variation of Ra can be obtained by varying the mean pressure. Note that varying the mean temperature will affect the radiation-conduction number $N_{r}$ while the variations of Ra were carried out at fixed $N_{r}$.

Once again, the computations start from a steady solution obtained at a moderate value of Rayleigh number $R a=10^{6}$ and $L=0.247 \mathrm{~m}$. The wall emissivity is assumed to be identical on all faces with value $\varepsilon=0.2$. By gradually increasing the Rayleigh number, the asymmetric steady solutions persist up to $R a=3.5 \times 10^{6}$ for which the flow is close to becoming unstable. One notable remark is that the more $R a$ is close to its critical value, the more the time becomes long for attaining the asymptotic solution. At $R a=4 \times 10^{6}$, the solution displays a periodic signal with a dimensionless frequency $f=0.3$. As expected, the surface radiation has a strong effect on the transition to unsteadiness. In Fig. 10(b), the extrapolated value of Ra corresponding to the transition to a periodic state is $R a_{c}=3.7 \times 10^{6}$. This critical value is much lower than in the case of pure convection which reveals that the surface radiation promotes the appearance of convective instabilities.

The critical value of $R a$ has been obtained for fixed values of cavity length and wall emissivity. Varying these parameters will affect the critical value $R a_{c}$ without changing the nature of the transition.

\subsubsection{Mean flow and temperature fluctuations}

3.2.2.1. Case with surface radiation. To examine the fluctuating fields, we have calculated the average flow by integrating the timedependent equations over a sufficiently long time and then computed the fluctuations by subtracting the mean solutions from

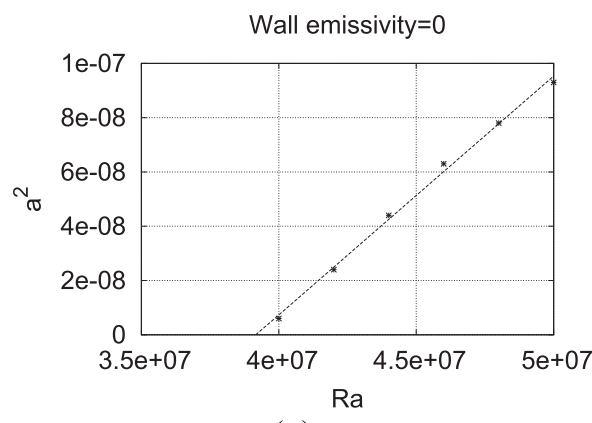

(a)

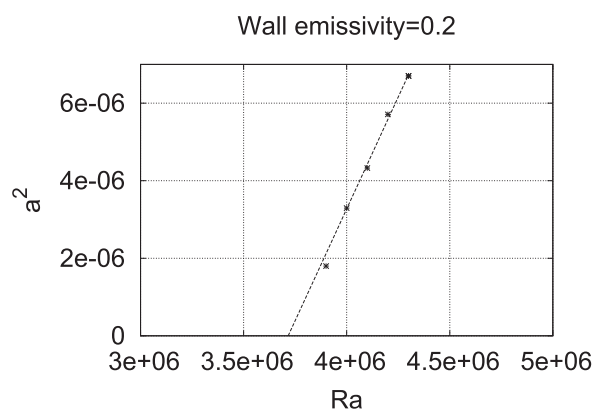

(b)

Fig. 10. Variation of squared amplitude of temperature vs Ra: (a) reflecting walls, (b) wall emissivity $\varepsilon=0.2$. 


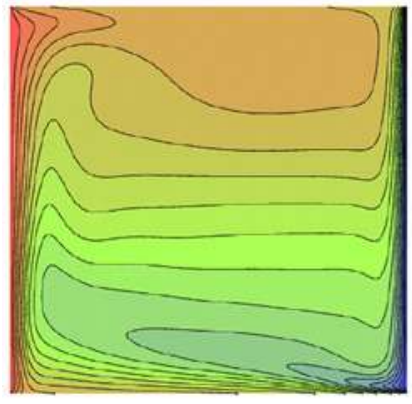

Mean temperature

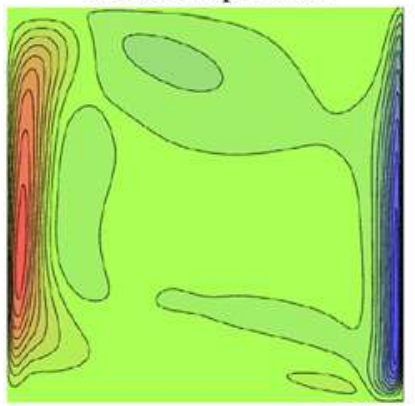

Mean $v_{z}$-velocity

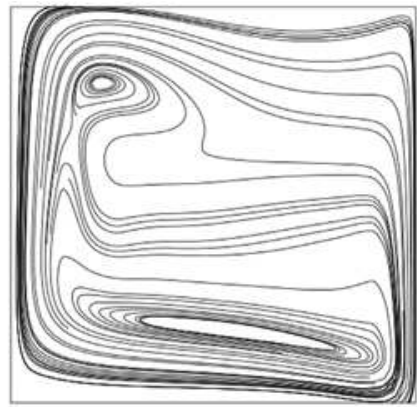

Mean stream function

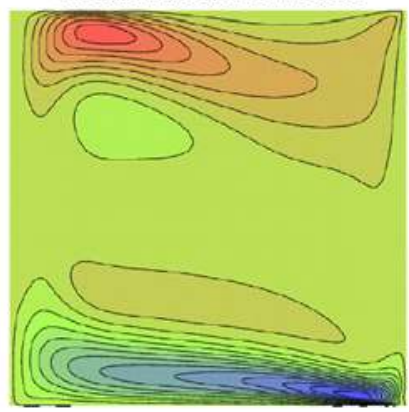

Mean $v_{x}$-velocity

Fig. 11. Contours of mean fields of temperature, stream function, velocities $v_{x}$ and $v_{z}$ for $R a=4 \times 10^{6}, \delta=0.6$ and wall emissivity $\varepsilon=0.2$.

instantaneous fields. Fig. 11 shows the mean fields of temperature, stream function and contours of mean velocity components for $R a=4 \times 10^{6}$. The mean flow is mainly dominated by a large clockwise cell with inside small rolls located near the bottom wall and at the top left corner of the cavity. The mean $v_{x}$-component show contours which develop smoothly parallel to the horizontal walls.

Fig. 12 shows snapshots of temperature fluctuations at different instants over one time period. These fluctuations occur as large

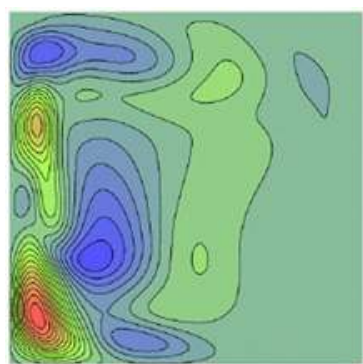

(a) $t_{0}$

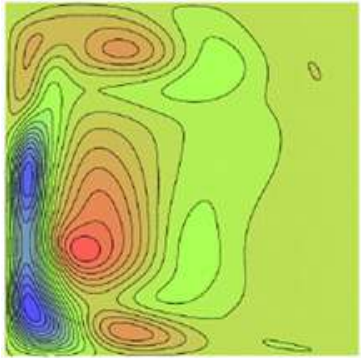

(e) $t_{0}+\frac{T}{2}$

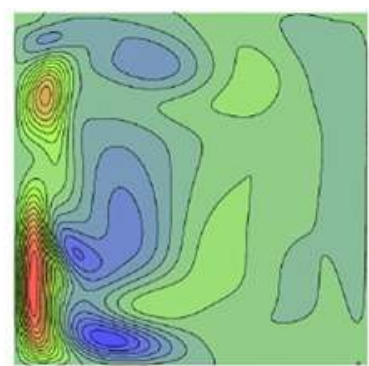

(b) $t_{0}+\frac{T}{8}$

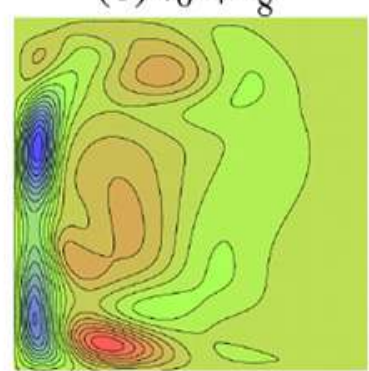

(f) $t_{0}+\frac{5 T}{8}$ structures with alternating sign which develop essentially on the left half of the cavity. A careful look to these plots indicates that the fluctuations with maximum intensity are concentrated near the walls and travel mainly in the shear layers. Since the boundary layer is much thicker on the hot wall than that on the cold wall for $\delta=0.6$, the fluctuations are convected along the hot wall and vanish on the cold boundary layer. A similar behavior has been observed by Le Quéré et al. [32] in a tall vertical cavity $(A=8)$ for large non-Boussinesq regimes $(\delta=0.6)$ in pure convection. In their previous work, the authors have shown the influence of the parameter $\delta$ on the structure of unsteady solutions. For low values of $\delta$, the fluctuations travel around the cavity and with increasing $\delta$, the instabilities are amplified in the upward boundary layer and damped as they travel down on the cold wall. Our numerical results confirm these observations by performing calculations with two other values of $\delta$ and maintaining the wall emissivity $\varepsilon=0.2$. In Fig. 13, are displayed spatial distributions of fluctuations for $\delta=0.05, \delta=0.2$ and $\delta=0.6$ at weak supercritical Rayleigh numbers corresponding to a periodic regime. For $\delta=0.05$ equivalent to a temperature difference $\Delta T=60 \mathrm{~K}$, alternate positive and negative fluctuations are distributed along the primary flow around the cavity walls. This scenario reproduces for $\delta=0.2$ $(\Delta T=240 \mathrm{~K})$ but with the alteration of fluctuations in the lower part of the cold wall.

Wang et al. [3] investigated the transition to unsteadiness in a two-dimensional square cavity with surface radiation under the Boussinesq approximation. They suggested that the mechanism leading to unsteady flow are caused by the onset of thermal instabilities like in cavity with perfectly conducting horizontal walls (linear temperature profile). The authors observed similar effects on temperature and flow fields between the cases: coupled convection-radiation and perfectly conducting lateral walls. It must be emphasized that the thermal instabilities related to conducting lateral walls result from an unstable stratification along the horizontal boundary layers (a stable stratification is a function of $z$ only) and are more destabilizing $\left(R a_{c}=2 \times 10^{6}[31]\right)$ than the hydrodynamic instabilities encountered in cavity with adiabatic horizontal walls $\left(R a_{c}=1.8 \times 10^{8}[31]\right)$.

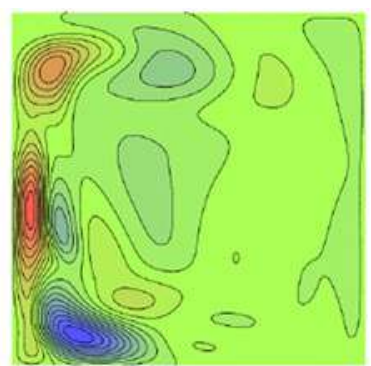

(c) $t_{0}+\frac{T}{4}$

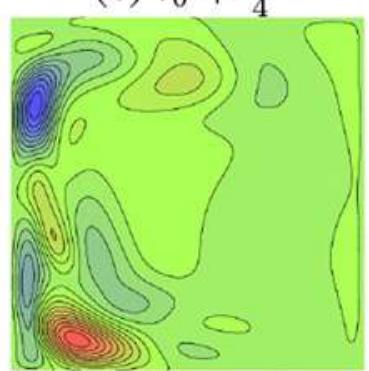

(g) $t_{0}+\frac{3 T}{2}$

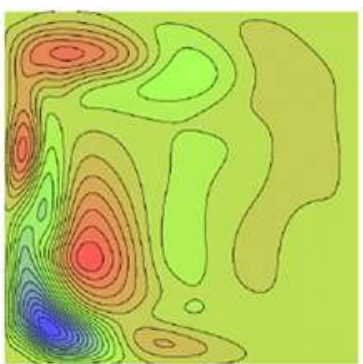

(d) $t_{0}+\frac{3 T}{8}$

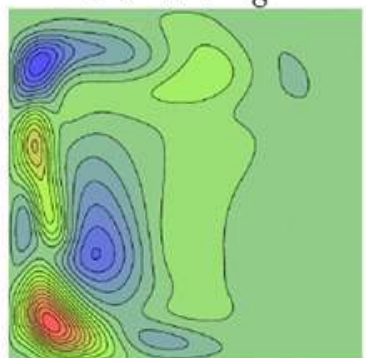

(h) $t_{0}+\frac{7 T}{8}$

Fig. 12. Snapshots of fluctuating fields of temperature at several instants over one period for $R a=4 \times 10^{6}$. 


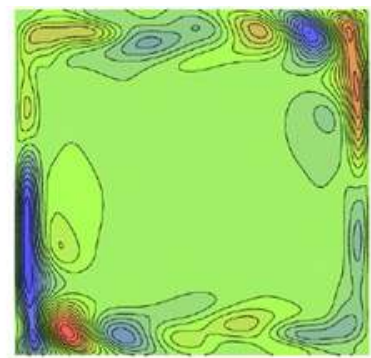

(a) $\delta=0.05 ; R a=8.5 \times 10^{6}$

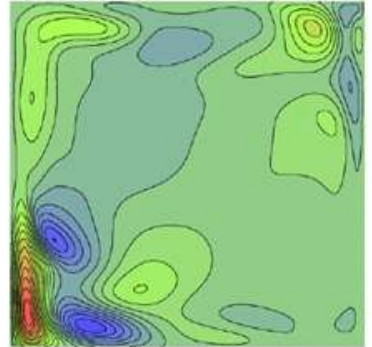

(b) $\delta=0.2 ; R a=9 \times 10^{6}$

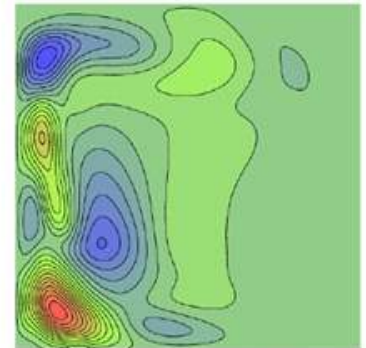

(c) $\delta=0.6 ; R a=4 \times 10^{6}$

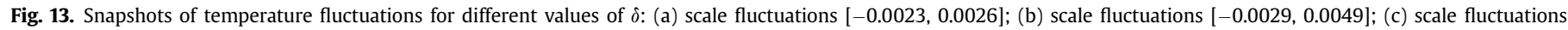
[-0.0211, 0.0218].

In this study, the origin of instabilities in natural convection combined to surface radiation are probably due to onset of thermal instabilities at the horizontal walls before traveling in the vertical boundary layers. The presence of unstable stratification zones near the horizontal walls and an earlier onset of instabilities compared to the case in pure convection are two relevant points to be considered. However, the spatial distribution of fluctuating fields is highly influenced by the strong non-Boussinesq regime. Only half of the cavity is concerned by these disturbances for $\delta=0.6$ while the distribution becomes more structured and covers all the cavity walls for $\delta=0.05$.

It is interesting to point out that the similarity observed by Wang et al. [3] between the coupled convection-radiation problem and the case with conducting horizontal walls, is not found when considering the non-Boussinesq regime. This behavior is due to the strong non-linear form of radiative heat flux when large temperature differences are considered. Note that in the Boussinesq approximation and at very low temperature differences, it is possible to approximate the dimensional net radiative flux by a linear expression in $\Delta T$ [3]. To evidence such effects, Fig. 14 displayed the temperature and flow fields obtained in a cavity with perfectly conducting horizontal walls and a cavity with four black

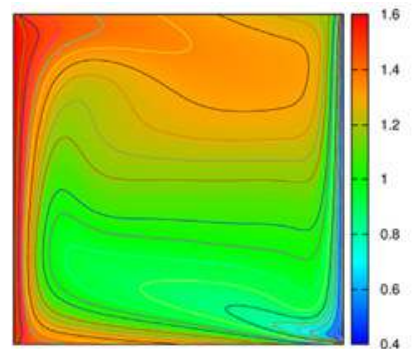

(a)

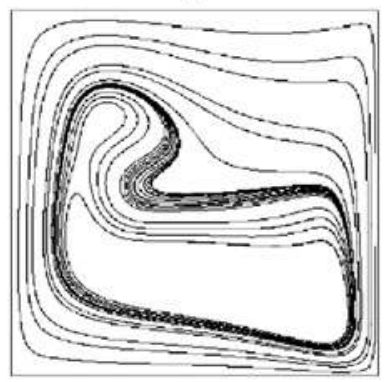

(a)

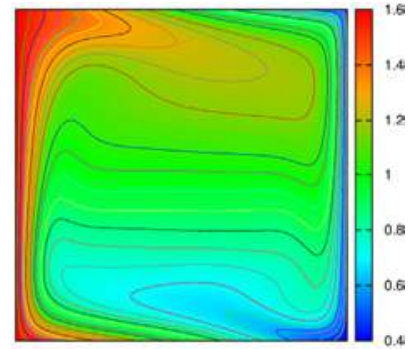

(b)

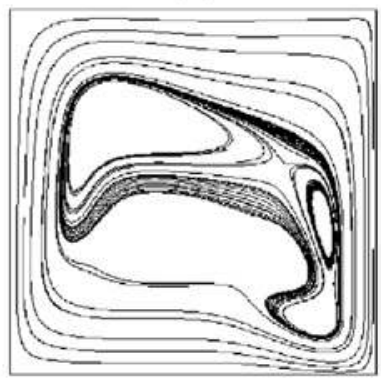

(b)
Fig. 14. Isotherms and streamlines for $R a=10^{6}$ : (a) cavity with four black walls; (b) cavity with perfectly conducting horizontal walls. walls at $R a=10^{6}$. The later configuration leads to almost similar temperature profiles at the top and bottom walls (see Fig. 3(b)). In both cases, it can be seen that the streamlines show peripheral flows which develop smoothly along the walls but exhibit quite different patterns at the cavity center. For conducting horizontal walls, spaced horizontal streamlines settle at the core with a small eddy located close to the top left corner. In the coupled convection-radiation case, the cavity core includes two small rolls at the top left corner and close to the bottom wall. The temperature fields present qualitatively some similarities: a stable thermal stratification at the core and unstable stratified zones on the horizontal walls. In the convection-radiation case, the unstable stratification is more developed at the bottom wall; the asymmetric distribution has been discussed previously. Considering the results, the presence of these unstable zones are probably at the origin of instability onset.

3.2.2.2. Case without surface radiation. Now compare the fluctuating fields with those obtained in pure convection at $R a=5 \times 10^{7}$. In Fig. 15, are displayed the mean fields of temperature, streamlines

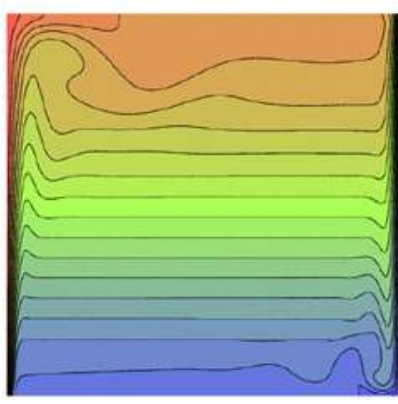

Mean temperature

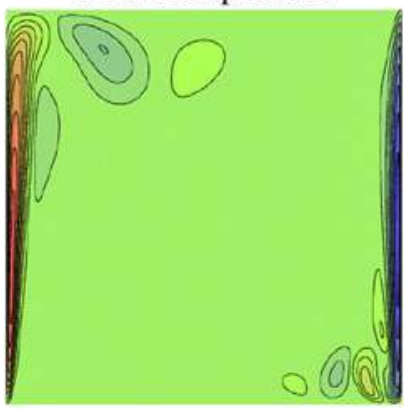

Mean $v_{z}$-velocity

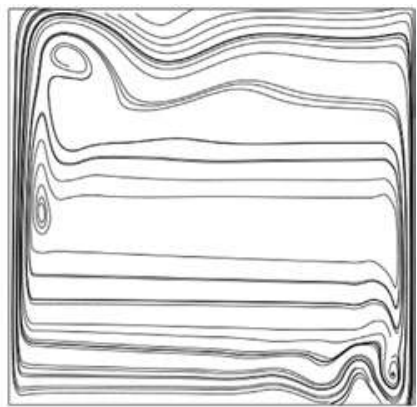

Mean stream function

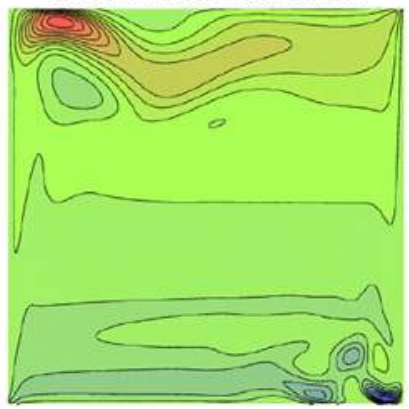

Mean $v_{x}$-velocity
Fig. 15. Contours of mean fields of temperature, stream function, velocities $v_{x}$ and $v_{z}$ for $R a=5 \times 10^{7}, \delta=0.6$ and wall emissivity $\varepsilon=0$. 


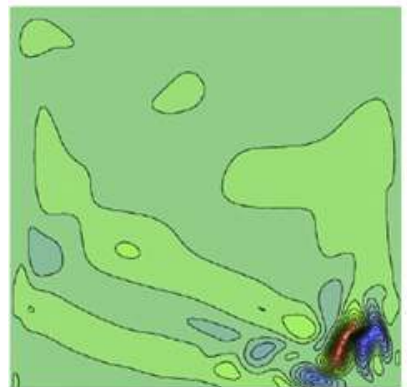

Temperature

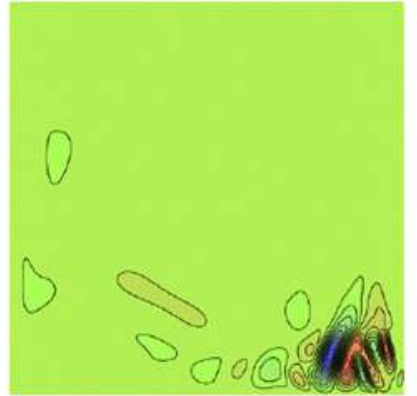

$v_{z}$-velocity

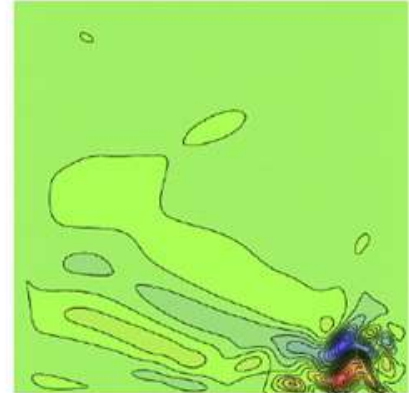

$v_{x}$-velocity

Fig. 16. Snapshots of fluctuating fields of temperature and velocity components for $R a=5 \times 10^{7}, \delta=0.6$ and wall emissivity $\varepsilon=0$.

and velocity components. The mean flow is characterized by a sudden expansion at the exit of vertical boundary layers causing the detachment of streamlines on the horizontal walls. Since the flow is asymmetric, the lower right corner of the cavity exhibits a rebound of the main stream more pronounced than the one on the top left corner. Unlike the precedent case, contours of the mean velocity $v_{x}$ are non smoothly parallel to the horizontal walls. As seen in Fig. 16, spatial distributions of temperature and velocity fluctuations highlight the presence of an unstable area only in the lower right corner. This behavior is due to nonlinear variations of transport coefficients with temperature near the cold wall which creates favorable conditions for the formation of disturbances. As the thermal conductivity and the viscosity decrease with temperature, the dissipation effects which are stabilizing are reduced at the right bottom corner and thus the disturbance structures appear.

There is no agreement in the literature for the physical mechanism of instabilities. In some previous studies, the corner flow was considered to be caused by a wave breaking of the internal hydraulic jump. This explanation was supported by Chenoweth and Paolucci [33] but refuted by Patterson and Armfield [34] and Ravi et al. [35]. The authors [35] argued that the flow separation at the corner is due to thermal effects. At high Rayleigh number, the relatively cold fluid in the upward vertical boundary layer detaches from the ceiling like a plume after impacting on the top corner. Note that the thermal stratification at the core is more important in pure convection. When the surface radiation is taken into account, the stratification becomes smaller, the velocities are intensified near the horizontal walls and the typical detached flow at the corners disappears.

Surface radiation is not the only parameter changing the instability mechanism. Transition to unsteadiness in natural convection flow induced by a large temperature difference $(\delta=0.6)$ has been carried out by Weissman et al. [30] in differentially heated cavities. The authors investigated the transition for different aspect ratios $(2 \leq A \leq 8)$ and have shown that the instability mechanisms depend strongly on this parameter.

\section{Conclusion}

Coupled natural convection and surface radiation is studied numerically in a differentially heated square cavity. The twodimensional flow is described by the Low Mach Number equations where nonlinear fluid properties and large temperature differences are taken into account. The heat transfer and flow characteristics are analyzed at steady state and the first transition from steady to unsteady flow is investigated for strong nonBoussinesq regimes. The influence of radiation on the flow structure and the heat transfer has been clearly established.
At steady state, the surface radiation reduces the convection heat transfer at the hot wall and increases it on the cold wall. The total heat transfer on the vertical walls (convection and radiation) increases with increasing $\varepsilon$ for identical wall emissivity on all faces. The surface radiation leads to the cooling of the top wall and the heating of the bottom wall. It has also be found that the temperature difference between the horizontal walls and the thermal stratification reduce with increasing the emissivity.

Transition to unsteadiness is investigated by increasing the Rayleigh number. The surface radiation has a non-stabilizing effect and leads to reduce the critical Rayleigh number by comparison with the case in pure convection. The flow undergoes a supercritical Hopf bifurcation in both cases without and with radiation but the instability mechanisms involved are different. In pure convection, the detached flow structure is responsible for the transition to a periodic motion. For the coupled convection-radiation problem, the instabilities are probably originated from the onset of thermal instabilities on the horizontal walls.

\section{Acknowledgments}

The authors are grateful to prof. Olivier Daube for his compressible code and for his helpful suggestions.

\section{References}

[1] M. Akiyama, Q.P. Chong, Numerical analysis of natural convection with surface radiation in a square enclosure, Numer. Heat Transf. A 32 (1997) 419-433.

[2] G. Colomer, M. Costa, R. Consul, A. Oliva, Three-dimensional numerical simulation of convection and radiation in a differentially heated cavity using the discrete ordinates method, Int. J. Heat Mass Transf. 47 (2004) 257-269.

[3] H. Wang, S. Xin, P. Le Quéré, Étude numérique du couplage de la convection naturelle avec le rayonnement de surfaces en cavité carrée remplie d'air, C. R. Mec. 334 (2006) 48-57.

[4] E.H. Ridouane, M. Hasnaoui, A. Campo, Effects of surface radiation on natural convection in a Rayleigh-Benard square enclosure: steady ans unsteady conditions, Heat Mass Transf. 42 (2006) 214-225.

[5] D. Iyi, R. Hasan, R. Penlington, Numerical analysis of the influence of thermal boundary condition and surface emissivity on the flow and heat transfer in turbulent buoyancy driven flow, in: Proceeding ASCHT, September 22-26, 2011. Kyoto Japan.

[6] A. Mezrhab, H. Bouali, H. Amoui, M. Bouzidi, Computation of combined natural-convection and radiation heat-transfer in a cavity having a square body at its center, Appl. Energy 83 (2006) 1004-1023.

[7] H. Sun, E. Chénier, G. Lauriat, Effect of surface radiation on the breakdown of steady natural convection flows in a square, air-filled cavity containing a centered inner body, Appl. Therm. Eng. 31 (2011) 1252-1262.

[8] M. Faghri, Y. Asako, G.M. Berard, A. Chaboki, Unsteady laminar natural convection, radiation and conduction within an enclosure with an obstruction, Int. J. Sci. Technol. 1 (1994) 85-95.

[9] G. Cesini, M. Paroncini, G. Cortella, M. Manzan, Natural convection from a horizontal cylinder in a rectangular cavity, Int. J. Heat Mass Transf. 42 (1999) 1801-1811. 
[10] H. Bouali, A. Mezrhab, H. Amaoui, M. Bouzidi, Radiation-natural convection heat transfer in an inclined rectangular enclosure, Int. J. Therm. Sci. 45 (2006) 553-566.

[11] M. Jami, M.A. Moussaoui, M. Mezrhab, D. Lemonnier, Modelisation du couplage convection naturelle rayonnement dans une cavite contenant un cylindre, J. Int. Therm. (Août 28-30, 2007). Albi France.

[12] S. Saravanan, C. Sivaraj, Coupled thermal radiation and natural convection heat transfer in a cavity with a heated plate inside, Int. J. Heat Fluid Flow 40 (2013) 54-64.

[13] H. Ambarita, K. Kishinami, M. Daimaruya, T. Saitoh, H. Takahashi, J. Suzuki, Laminar natural convection heat transfer in an air filled square cavity with two insulated baffles attached to its horizontal walls, Therm. Sci. Eng. 14 (2006) 35-46.

[14] M. Rabhi, H. Bouali, A. Mezrhab, Radiation-natural convection heat transfer in inclined rectangular enclosures with multiple partitions, Energy Convers. Manag. 49 (2008) 1228-1236.

[15] G. Lauriat, G. Desrayaud, Effect of surface radiation on conjugate natural convection in partially open enclosures, Int. J. Therm. Sci. 45 (2006) 335-346.

[16] A. Mezrhab, S. Amraqui, C. Abid, Modelling of combined surface radiation and natural convection in a vented "T" form cavity, Int. J. Heat Fluid Flow. 31 (2010) 83-92.

[17] A. Bahlaoui, A. Raji, M. Hasnaoui, C. Ouardi, M. Naïmi, T. Makayssi, Height partition effect on combined mixed convection and surface radiation in vented rectangular cavity, J. Appl. Fluid Mech. 4 (2011) 89-96.

[18] R. Li, M. Bousetta, E. Chénier, G. Lauriat, Effect of surface radiation on natural convective flows and onset of flow reversal in asymmetrically heated vertica channels, Int. J. Therm. Sci. 65 (2013) 9-27.

[19] A. Raji, M. Hasnaoui, S. Goujon-Durand, P. Vasseur, Numerical study of the effect of the insulation of the walls on natural convection in a differentially heated cavity, ISJAEE 6 (2008) 122-130.

[20] N.Y. Zhan, M. Yang, P.W. Xu, A two-dimensional study on natural convection and heat transfer in the enclosure with heat transfer and radiation coupled in natural convection, Sci. China Tech. Sci. 53 (2010) 991-999.

[21] S. Xin, J. Salat, P. Joubert, A. Sergent, F. Penot, P. Le Quéré, Resolving the stratification discrepancy of turbulent natural convection in differentially heated air-filled cavities. Part III: a full convection-conduction-surface radiation coupling, Int. J. Heat Fluid Flow. 42 (2013) 33-48.

[22] I. Teleaga, M. Seaïd, I. Gasser, A. Klar, J. Struckmeier, Radiation models for thermal flows at low Mach number, J. Comput. Phys. 215 (2006) 506-525.
[23] B. Dubroca, M. Seaïd, I. Teleaga, A consistent approach for the coupling of radiation and hydrodynamics at low mach number, J. Comput. Phys. 225 (2007) 1039-1065.

[24] G. Scarella, G. Accary, S. Meradji, D. Morvan, O.A. Bessonov, Threedimensional numerical simulation of the interaction between natural convection and radiation in a differentially heated cavity in the low mach number approximation, in: ICHMT International Symposium on Advances in Computational Heat Transfer, May 11-16, 2008. Marrakesh Morocco.

[25] S. Paolucci, On the Filtering of Sound from the Navier-Stokes Equations, Sandia National Laboratory Report SAND 82-8257, 1982, pp. 3-52.

[26] M. Bouafia, O. Daube, Natural convection for large temperature gradient around a square solid body within a rectangular cavity, Int. J. Heat Mass Transf. 50 (2007) 3599-3615.

[27] J.R. Howell, A Catalog of Radiation Configuration Factors, McGraw-Hill, New York, 1982

[28] P. Le Quéré, C. Weisman, H. Paillère, J. Vierendeels, E. Dick, R. Becker M. Braack, J. Locke, Modelling of natural convection flows with large temperature differences: a benchmark problem for low Mach number solvers. Part 1. Reference solutions, M2AN 39 (2005) 609-616.

[29] P. Le Quéré, Accurate solution in the square thermally driven cavity at high Rayleigh number, Comput. Fluids 20 (1991) 29-41.

[30] C. Weisman, D. Barkley, P. Le Quéré, Transition to unsteadiness of nonBoussinesq natural convection solutions, in: I.C.C.H.M.T, May 10-17, 2005. Cachan France.

[31] P. Le Quéré, Etude de la transition à l'instationnarité des écoulements de convection naturelle en cavité verticale différentiellement chauffée par méthodes spectrales Chebyshev, Thèse d'Etat, Université de Poitiers, France, 1987.

[32] P. Le Quéré, R. Masson, P. Perrot, A chebyshev collocation algorithm for 2D non-Boussinesq, J. Comput. Phys. 103 (1992) 320-335.

[33] D.R. Chenoweth, S. Paolucci, Natural convection in an enclosed vertical air layer with large horizontal temperature differences, J. Fluid Mech. 169 (1986) $173-210$.

[34] J.C. Patterson, S.W. Armfield, Transient features of natural convection in a cavity, J. Fluid Mech. 219 (1990) 469-497.

[35] M.R. Ravi, R.A.W.M. Henkes, C.J. Hoogendoorn, On the high-Rayleigh-number structure of steady laminar natural-convection flow in a square enclosure, J. Fluid Mech. 262 (1994) 325-351. 